\title{
Trends in mental health system transformation: Integrating youth services within the Canadian context
}

\section{Tanya Halsall $^{1,2}$; Ian Manion ${ }^{1,2,3}$; Srividya N. Iyer $^{2,4,5,6}$; Steve Mathias ${ }^{2,7,8}$; Rosemary Purcell ${ }^{2,9,10}$; and Joanna Henderson ${ }^{2,11,12}$}

1 The Royal's Institute of Mental Health Research, Ottawa, Ontario, Canada.

2 Frayme, International Knowledge Translation Platform, Ottawa, Ontario, Canada.

3 School of Psychology, University of Ottawa, Ottawa, Ontario, Canada.

4 ACCESS Open Minds (pan-Canadian youth mental health research network), Montreal, Quebec, Canada.

5 Douglas Mental Health University Institute, Montreal, Quebec, Canada.

6 Department of Psychiatry, McGill University, Montreal, Quebec, Canada.

7 Foundry, Vancouver, British Columbia, Canada.

8 Department of Psychiatry, University of British Columbia, Vancouver, British Columbia, Canada.

9 Orygen, National Centre of Excellence in Youth Mental Health, Parkville, Victoria, Australia.

10 Centre for Youth Mental Health, The University of Melbourne, Parkville, Victoria, Australia.

11 Margaret and Wallace McCain Centre for Child, Youth and Family Mental Health, Toronto, Ontario, Canada.

12 University of Toronto, Centre for Addiction and Mental Health, Toronto, Ontario, Canada.

\section{Corresponding author:}

Tanya Halsall, University of Ottawa Institute of Mental Health Research, Ottawa, Ontario, Canada.

E-mail: tanya.halsall@theroyal.ca

Halsall, T., Manion, I., Iyer, S. N., Mathias, S., Purcell, R., \& Henderson, J. (2019). T rends in mental health system transformation: Integrating youth services within the Canadian context . Healthcare Management Forum, 32(2), 51-55. https://doi.org/10.1177/0840470418808815 Cpyright (C) 2015 (Sage). 


\begin{abstract}
The current mental health services system in Canada is fragmented and transitions between the youth and adult mental health systems have been identified as needing significant improvement. Integrated youth services (IYS) are designed to be adaptable and developmentally appropriate as well as to promote seamless transitions, including during emerging adulthood. This paper provides an overview of recent developments in Canadian mental health system transformation to promote the integration of services and the holistic promotion of youth wellbeing. We offer an overview of the current state of knowledge related to best practices in IYS in Canada and highlight areas for future development. We also introduce Frayme, a Canadian-based international knowledge translation platform designed to connect organizations working in the youth services system to accelerate the implementation of IYS.
\end{abstract}

\title{
Introduction
}

Adolescence and emerging adulthood are crucial stages that influence developmental trajectories over the lifespan. ${ }^{1}$ As modern society has become increasingly complex, researchers argue that there is a widening gap between the dependence of childhood and the responsibilities of adulthood and a scarcity of incremental opportunities to promote transition. ${ }^{2-4}$ During this time, youth are at increased risk of developing mental health issues with approximately $75 \%$ of lifetime adult mental disorders commencing before the age of $18 .^{5}$ In Canada, youth aged 15 to 24 report the highest rates of mood disorders (8.2\%) and substance use disorders $(11.9 \%)$ compared with other age groups. ${ }^{6}$ Furthermore, suicide is the second leading cause of death in Canadian young people. ${ }^{7}$ As important as this period of development appears to be for the emergence of mental health issues, it is at this juncture where our systems of care arguably show their greatest weaknesses. ${ }^{8}$ 
The current mental health services system in Canada is fragmented ${ }^{9-13}$ and transitions between the youth and adult mental health systems have been identified as needing significant improvement. ${ }^{8,9,14}$ Services are characterized by delays in providing appropriate care, a lack of youth and family engagement and silos based on diagnosis prompting service users, thought leaders and academics ${ }^{15}$ to call for system transformation that increases integration of services and facilitates access to timely supports. "Services should address the individual needs of the help seeker, based on a lifespan-oriented approach. For youth and young adults, this includes a move towards maturity and independence, stable self-identity, peer relationships, economic independence, and recognition of physical, psychological and social changes ${ }^{9}$ (p. 20). Integrated youth services (IYS) are designed to be adaptable and developmentally appropriate as well as to

promote seamless transitions, including during emerging adulthood. ${ }^{14,16,17}$ This paper provides an overview of recent developments in Canadian mental health system transformation to promote the integration of services and the holistic promotion of youth wellbeing. We offer an overview of the current state of knowledge related to best practices in IYS in Canada and highlight areas for future development.

\section{Integrated youth services (IYS) initiatives in Canada}

Generally, IYS models aim to bring a range of services used by young people together, typically primary care, mental health, addictions, vocational and other social services to provide holistic, client-centered care ${ }^{11,14,17,18}$ with important links to other sectors such as education. IYS is not yet well-defined but refers to initiatives with a core set features and a commitment to service integration. In describing a model as an "IYS" we do not assume that each approach is the same or that the providers ascribe to the term themselves, but that at the very least, they cater to youth and have at least a few different services that are beneficial to youth. 
In other global contexts, nation-wide transformations have been implemented within Australia $^{19}$ (headspace) and Ireland ${ }^{20}$ (Jigsaw), whereas in Canada, IYS models have yet to be widely adopted. In part, this is a reflection of having health funding and services organized by province and territory rather than federally. Furthermore, in Canada as in other nations, we must consider diversity, including cultural, geographic, linguistic and level of capacity. There is also a need for special consideration of Indigenous communities as these contexts reflect significant diversity and represent unique strengths and challenges.

Current initiatives. Recent uptake of IYS in Canada has largely been stimulated by government and philanthropic efforts. For example, in 2014, the Canadian Institutes of Health Research and the Graham Boeckh Foundation collaborated to launch the Transformational Research in Adolescent Mental Health (TRAM) initiative. This was the first of the Strategy for Patient-Oriented Research programs, providing \$25 million over five years to foster collaboration, and placing youth and their families at the centre of IYS. ACCESS Open Minds emerged from TRAM to develop a pan-Canadian research and evaluation network with 14 sites within six provinces and one territory. Subsequently, the Graham Boeckh Foundation partnered with the St Paul's Foundation and the government of British Columbia to create Foundry IYS. Originating from Vancouver's Inner City Youth program, Foundry has since opened seven centres in British Columbia with plans for four more. In Ontario, several IYS centres have developed out of recognized need without connection to a broader initiative. YouthCan IMPACT (Toronto) is a pragmatic research and implementation trial involving the development and implementation of three IYS hubs in Toronto, Ontario. ACCESS Open Minds also supports a site operating in Ontario More recently, the government of Ontario provided funding to ten Youth Wellness Hubs Ontario (YWHO) sites and provided a funded backbone support to align sites 
into a provincial network. The government of New Brunswick initiated three of the ACCESS Open Minds demonstration sites as part of a planned rollout of IYS services province-wide. Encouragingly, there are plans for adoption of IYS in the provinces of Alberta, Quebec, Manitoba, Newfoundland as well as the Northwest Territories. Internationally, research has identified that the IYS approach is effective in increasing access to vulnerable youth populations (Hilferty, 2015; O’Keefe, 2015). Researchers identified that youth participants at Jigsaw exhibited significant reductions in psychological distress (O’Keefe, 2015). The Headspace program in Australia demonstrated similar results when compared to a control group and another treatment group (Hilferty, 2015). In Canada, studies examining the effectiveness of IYS are ongoing, including a pragmatic randomized controlled trial (YouthCAN Impact) and a research and evaluation initiative examining sites across the country (ACCESS Open Minds).

\section{Major IYS components and future directions}

Operational structures. To support the growth of IYS nationally, leaders in the field are coming together to identify fundamental structures and processes required to successfully implement an IYS. Elements under discussion include operational logistics, governance structures, communication strategies, policy development, youth-friendly space design and data management, to name a few. Additional considerations include the incorporation of evidencebased practices, application of stepped-care models, scalable implementation and rigorous evaluation. The meaningful engagement of youth and families, and the incorporation of perspectives from diverse populations represented within the community have been deemed paramount.

Above all, the development of IYS requires the building of positive relationships. In support of creating and sustaining partnerships, there are several essential components including 
the creation of a technical assistance or "backbone" body (e.g. Foundry, ACCESS Open Minds and YWHO). In other jurisdictions (e.g., New Brunswick), a governance committee serves a key function in overseeing the implementation of the IYS. ${ }^{21}$ These bodies oversee the development of operational and system policies to support the alignment of IYS sites with guiding principles and overall strategy. The bodies have created data systems to support data sharing while ensuring privacy and confidentiality. In addition, and unique to this sector, well-designed brands, with a common understanding among partners and the promotion of youth and family awareness and participation ${ }^{17,21}$ within a marketing and communications strategy is viewed as critical.

Services. Experts have identified that there is a need for more research and evaluation of IYS generally. ${ }^{14}$ Researchers have identified that IYS programs need to be better described. ${ }^{17}$ One direction is to examine services and skills essential for IYS. ${ }^{17}$ As aforementioned, primary care, mental health, addictions, vocational and other social services are typically offered within an IYS complement. ${ }^{18}$ Specific evidence-based practices applied within IYS contexts have been identified, including: Solution-Focused Brief Therapy, Motivational Interviewing, TraumaFocused Cognitive Behavioral Therapy, Dialectical Behaviour Therapy, and Mindfulness ${ }^{14,22}$ yet it is unknown what the minimum services and components are to establishing an effective IYS. Future research should seek to identify these critical components by cross-comparing processes, model implementation and measured impact. These examinations will also need to identify what core skills and competencies are necessary for IYS. An already identified attribute of the delivery of IYS is the presence of a general affinity for young people ${ }^{14,17}$, yet this attribute is often experienced as lacking by youth in their service providers. ${ }^{23,24}$ The implication is that agencies need to create policies, as well as training and recruitment strategies to hire appropriate, youth-positive staff. ${ }^{14}$ Evaluations should also examine cost-benefit/cost-effectiveness ratios as, 
to date, only one published examination of return on investment exists. An investigation of Headspace adaptation in Denmark found that a 2.3 million DKK (475,000 CAD) investment resulted in a13.9 million DKK (2.9 million CAD) return. ${ }^{25}$ Currently in Canada, a number of comprehensive evaluations are underway.

Processes. The utilization of stepped-care models and the development of clinical pathways are significant tools for facilitating services within IYS. Stepped-care models organize treatment options hierarchically by intensity and base treatment decisions on specific criteria. ${ }^{26}$ Pathways map out critical clinical and administrative processes to organize the range of services and providers involved in a youth's care. ${ }^{14}$ In future, it will be important to examine pathways that incorporate educational settings (i.e. high schools, post-secondary institutions) both to step up (promotion, prevention, early identification, stigma reduction) and step down from clinical services. Placing IYS services in schools and post-secondary institutions can help shift the focus to promotion, prevention and early identification ${ }^{9,21}$ and has been identified as a viable way to decrease waitlists and to expand beyond formalized services to other developmental contexts such as moving services into the home and community. ${ }^{21}$

One of the access challenges that has been identified within the youth services literature is providing convenient service hours. ${ }^{14,17}$ Complementary e-delivery of services is increasingly considered feasible ${ }^{9}$ as remote access overcomes a lack of local services.

Peer, youth and family engagement. Stigma has been identified as an on-going issue that can affect access. ${ }^{17}$ Recognizing that lived experience and knowledge of coping with related mental health challenges are unique contributions provided by peer support workers in mental health services, peer support programs can create positive change through the reduction of stigma. ${ }^{27-29}$ Social norms within peer support programs that emphasize inclusion as well as a 
strengths-based focus on agency and empowerment may help to reduce barriers to access and engagement created by stigma, while facilitating acceptance, engagement and coping. Stigma, however, has also been identified as an on-going issue for peer support workers within the organizational environment and this should be examined in future research. ${ }^{28}$

Examinations of waitlists in IYS have identified positive and negative results. Within a review of IYS models, one of the limitations identified was that high demand resulted in long waitlists ${ }^{17}$. In contrast, within another report, school-based preventative approaches have demonstrated decreased waiting lists ${ }^{21}$. Identifying what factors and processes decrease waitlists and promote accessibility within IYS should be a goal moving forward. The evidence for schoolbased IYS is still limited and not well-integrated.

A fundamental contribution of IYS models is the inclusion of meaningful youth and family engagement processes. Youth engagement has been identified as integral to four IYS components: the planning and design of IYS sites and services, the promotion of relationships between youth and staff, the empowerment of youth within their own treatment plan and within peer support services. ${ }^{14}$ Application of youth engagement in design and an emphasis on strengths-based approaches may also be viable ways to overcome stigma. There is a need to better understand individual outcomes for youth who are formally engaged in mental health governance. Researchers have often emphasized the significance of taking a long view when focusing on skill building with youth. ${ }^{9,17}$ Recognizing that these youth soon transition to adulthood, it is imperative to understand how they apply these skills as they advance in their careers and to learn whether stigma or stigma reduction through engagement has an impact on their trajectory. Facilitating the inclusion of families can support continuity of care, improve transitions, promote recovery, enhance youth wellbeing and increase family functioning ${ }^{9}$, 
however, there is much less known about how to engage family within IYS. Learning how to empower families in a cohesive way alongside youth without inhibiting youth independence is an area that is not yet well-understood.

Policy. A key insight relates to the need to go beyond programming and operational transformation and place a focus on policy. Funding priorities and political structures can facilitate or inhibit the development of partnerships, collaborative work and sustainability. Organizations that are interested in pursuing IYS models must also recognize the political structures that may challenge their work and advocate to change them. For example, within New Brunswick's provincial-level IYS transformation, legislation was introduced to allow services to share information in order to determine program suitability before contacting clients regarding participation $^{21}$. These changes enabled youth and families to transition through services without having to continually retell their stories and supported the provision of services suitable to the level of need within the school and community context. Agencies that have a lead role in coordinating the delivery of IYS services, typically non-profit organizations, have identified a need to develop expertise in building and maintaining relationships ${ }^{14}$ within their community, as each community varies considerably and has existing relationships, services and structures that can be leveraged to create IYS. E-services and school-based liaisons will serve as effective complements for community-based access.

In addition to policy, there is a need for implementation tools that can be applied across contexts, such as community readiness assessments, common care plan templates, clinical pathways, memorandums of agreement, data-sharing software, standards of youth and family engagement and standardized measures. As such, implementation tools such as the template for 
intervention description and replication ${ }^{29}$ (TIDieR) may be useful to capture systematic information about the range of models.

\section{Frayme: An International Knowledge Translation Platform}

A recent initiative is taking these issues into consideration and providing support to organizations looking to implement IYS both in Canada and abroad. Frayme is a Canadian-based international knowledge translation platform funded by the Networks of Centres of Excellence supplemented with significant support from network partners and the philanthropic sector that connects organizations working in youth services systems to accelerate the implementation of IYS (frayme.ca). Currently, the Frayme network includes over 250 partners from eleven countries working within research, practice and government. The Frayme strategy is focused on integrated knowledge mobilization, including the synthesis of evidence, knowledge translation and the provision of implementation supports. Frayme seeks to foment the creation of political environments, knowledge supports and implementation tools that are needed to promote the uptake and scaling of IYS across Canada and the world. In the Canadian context, Frayme and its partners are already informing the rapid adoption of IYS. Health leaders with an interest in promoting integration between youth services can look to Frayme as a resource for leading edge knowledge, implementation tools and to connect to other partners working to advance the field.

In many ways, Frayme is becoming the adhesive between the various IYS initiatives in Canada, fostering mutual learning, co-creations of processes and products, and alignment of efforts as this model evolves nationally and beyond. Frayme accomplishes this while also rolemodelling partnership, co-creation and meaningful youth and family engagement. For example, in the spring of 2017, Frayme brought together over 60 partners from across Canada form policy, practice, research and mental health advocacy, including youth and family (Blind for Review). 
At this event, partners worked together to co-create Frayme priorities and inform future strategies to influence youth mental health services. Recognizing that there is a need for more clarity regarding core components in IYS, Frayme is also conducting an international scan to identify best practices and learn about the range of models and processes being implemented. Finally, Frayme has developed a youth advisory committee and a family advisory committee in order to help guide there strategic plans as well as to mobilize strategic projects within youth and family advocacy.

\section{Conclusion}

This paper provides an overview of recent developments within the Canadian youth mental health system and describes the current state of knowledge related to best practices in IYS. Partners are coming together in an unprecedented manner to generate a movement to address system fragmentation that includes the application of evidence as well as considerations related to the complexity of the implementation and community contexts. This represents a shift towards perceiving youth holistically by addressing their full range of needs and strengths to promote their development and to invest in the future of Canada. Evidence is emerging on core process and content elements that may be critical to the implementation and scalability of IYS within various Canadian contexts. Evidence for the outcomes of these elements taken together is still emerging. There is clear value in aligning the emerging efforts toward implementing IYS, evaluating process and outcomes with common metrics, and sharing this growing evidence base to further inform police and practice. The Frayme network represents an ideal vehicle to accomplish many of these goals. 


\section{Acknowledgements}

This review was supported by The Networks of Centres of Excellence, The Royal's Institute of Mental Health Research affiliated with the University of Ottawa and the Social Sciences and Humanities Research Council. 


\section{References}

1. Patton GC et al. Our future: a Lancet commission on adolescent health and wellbeing. The Lancet. 2016; 387, 2423-2478.

2. Benson PL. All kids are our kids: What communities must do to raise caring and responsible children and adolescents. San Francisco, CA: Jossey-Bass; 2006.

3. Eccles JS \& Gootman JA. Community programs to promote youth development. Washington, DC: National Academy Press; 2002.

4. Larson RW. Toward a psychology of positive youth development. Am. Psychol. 2000; 55, $170-183$.

5. Kessler RC, Berglund P, Demler O, Jin R, Merikangas KR, Walters EE. Lifetime prevalence and age-of-onset distributions of DSM-IV disorders in the National Comorbidity Survey Replication. Arch Gen Psychiatry. 2005;62(6):593-602.

6. Pearson C, Janz, T \& Ali J. Mental and substance use disorders in Canada. (Statistics Canada Ottawa, Ontario, 2012).

7. Bennett $\mathrm{K}$ et al. A youth suicide prevention plan for Canada: a systematic review of reviews. Can. J. Psychiatry. 2015; 60, 245-257.

8. Carver J, Cappelli M \& Davidson S. Taking the next step forward: Building a responsive mental health and addictions system for emerging adults. Mental Health Commission of Canada/ Commission de la santé mentale du Canada. 2015.

9. Provincial Territorial Working Group. Towards integrated primary and community mental health and substance use care for youth and young adults: A compendium of current Canadian initiatives and emerging best practices. 2016. 
10. Canadian Association of Paediatric Health Centres, The National Infant, Child, and Youth Mental Health Consortium Advisory, and The Provincial Centre of Excellence for Child and Youth Mental Health at CHEO. Access \& Wait Times in Child and Youth Mental Health: A Background Paper. The Provincial Centre of Excellence for Child and Youth Mental Health at CHEO. 2010.

11. Henderson JL, et al. Integrated collaborative care teams to enhance service delivery to youth with mental health and substance use challenges: protocol for a pragmatic randomised controlled trial. BMJ Open. 2017;7:e014080.

12. Kirby MJ, Keon WJ \& Dinsdale HB. Out of the shadows at last: Transforming mental health, mental illness and addiction services in Canada (Final Report of The Standing Senate Committee on Social Affairs, Science and Technology). 2006.

13. Waddell C, McEwan K, Shepherd CA, Offord DR \& Hua JM. A public health strategy to improve the mental health of Canadian children. Can. J. Psychiatry. 2005; 50, 226-233.

14. Salt V, Parker N, Ramage K \& Scott C. Community-based Mental Health Services Hubs for Youth Environmental Scan. (PolicyWise for Children \& Families, 2017).

15. Malla A, et al. Youth mental health should be a top priority for health care in Canada. Can J Psychiatry. 2018;63:216-222.

16. Halsall T, Manion IG, Henderson JL. Examining integrated youth services using the bioecological model: Alignments and opportunities. Int J Integr Care. 25(submitted).

17. Hetrick SE, et al. Integrated (one-stop shop) youth health care: best available evidence and future directions. Med J Aust. 2017; 207:5-18. 
18. Settipani CA, Cleverley K, Hawke LD, Rice M, Henderson JL. Essential components of integrated care for youth with mental health and addiction needs: protocol for a scoping review. BMJOpen. 2017;7:e015454.

19. Hilferty F, Cassells R, Muir K, Duncan A, Christensen D, Mitrou F, Gao G, Mavisakalyan A, Hafekost K, Tarverdi Y, Nguyen H, Wingrove C \& Katz I. Is headspace making a difference to young people's lives? Final Report of the independent evaluation of the headspace program. Social Policy Research Centre, 2015.

20. O'keeffe L, O’Reilly A, O’Brien G, Buckley R \& Illback R. Description and outcome evaluation of Jigsaw: an emergent Irish mental health early intervention programme for young people. Ir. J. Psychol. Med. 2015; 32, 71-77.

21. Morrison B, Peterson P \& Health and Education Research Group. Story of transformation in youth mental health in the province of New Brunswick. University of New Brunswick, 2017.

22. Henderson, JL, Cheung, A, Cleverley, K, Chaim, G, Moretti, ME, de Oliveira, C, ... \& Herzog, T. Integrated collaborative care teams to enhance service delivery to youth with mental health and substance use challenges: Protocol for a pragmatic randomised controlled trial. BMJ open, 2017; 7(2), e014080.

23. Brownlie EB, Chaim G, Heffernan O, Herzog T, Henderson J. Youth services system review: moving from knowledge gathering to implementation through collaboration, youth engagement, and exploring local community needs. Can J Commun Ment Health. 2017;36(Special Issue):133-149.

24. Chaim G, Henderson J, Brownlie EB. Youth Services System Review: A Review of the Continuum of Ontario Services Addressing Substance Use Available to Youth Aged. Toronto, ON: Centre for Addiction and Mental Health; 2013:12-24. 24. 
25. headspace Denmark. Economists Without Borders: headspace is a beneficial investment. (https://www.headspace.dk/en/about-us/documentation-and-reports/economists-withoutborders; 2017).

26. O'Donohue WT \& Draper C. The case for evidence-based stepped care as part of a reformed delivery system. in Stepped care and e-health: Practical applications to behavioral disorders 1-16 (New York: Springer Science + Business Media; 2011.

27. Alvarez-Jimenez M, Gleeson JF, Rice S, Gonzalez-Blanch C \& Bendall S. Online peer-topeer support in youth mental health: seizing the opportunity. Epidemiol. Psychiatr. Sci. 2016; $25,123-126$.

28. Barton J \& Henderson J. Peer Support and Youth Recovery: A Brief Review of the Theoretical Underpinnings and Evidence. Can. J. Fam. Youth J. Can. Fam. Jeun. 2016; 8, 117.

29. Blixen C et al. Training peer educators to promote self-management skills in people with serious mental illness (SMI) and diabetes (DM) in a primary health care setting. Prim. Health Care Res. Dev. 2015; 16, 127-137.

28. Delman J \& Klodnick VV. Factors supporting the employment of young adult peer providers: Perspectives of peers and supervisors. Community Ment. Health J. 2017; 53, 811822.

29. Hoffmann TC et al. Better reporting of interventions: template for intervention description and replication (TIDieR) checklist and guide. BMJ. 2014; 348, g1687. 